\title{
Nanosuspension: Potential Applications of Nano Therapeutics in Ocular Delivery
}

\author{
Nangare KA ${ }^{1}$, Powar SD ${ }^{1}$, Kate VK² ${ }^{2}$ Khavane $\mathrm{KK}^{2}$ and Payghan SA ${ }^{1 *}$ \\ ${ }^{1}$ Department of Pharmaceutics, Tatyasaheb Kore College of Pharmacy, India \\ ${ }^{2}$ Department of Pharmacy Practice \& Pharmacology, Rajesh Bhaiyya Tope College of Pharmacy, India
}

Submission: January 03, 2018; Published: February 20, 2018

*Corresponding author: Santosh Ambadas Payghan, Department of Pharmaceutics, Tatyasaheb Kore college of Pharmacy, Warananagar, Panhala, Kolhapur-416113, MS, India, Tel: +919096202858; Email: sapayghan.tkcp@gmail.com

\begin{abstract}
Nanotechnology involves creating and using the materials, devices or systems on the nanometer scale respectively. Nanotechnology is the science that deals with the process that occurs at molecular level and of nano length scale size. Nano refers to the particle size range of 1-1000nm. Nanosuspensions are coming under nanotechnology. The major challenge faced by today's pharmacologist and formulation scientist is ocular drug delivery. In the past two decades, ocular drug delivery research accelerated advanced towards developing a novel, safe and patient compliant formulation and drug delivery devices/techniques, which may surpass these barriers and maintain drug levels in tissues. These novel devices and/or formulations may help to surpass ocular barriers and associated side effects with conventional topical drops. The particle size influences its functionality in terms of its uptake, residence in circulation, adherence, degradation, clearance. This review article describes considerations of nanosuspension for ocular delivery, ocular barriers, strategies to overcome blood ocular barriers, ocular drug-delivery mechanisms, ocular pharmacotherapy, conventional pharmacotherapy of ocular diseases, and applications of nanosuspensions in ocular delivery.
\end{abstract}

Keywords : Nanosuspensions; Ocular barriers; Mechanisms; Pharmacotherapy; Application

\section{Introduction}

Nanotechnology involves creating and using the materials, devices or systems on the nanometer scale respectively. These technologies are expected to play a critical role in number of biomedical applications like drug delivery, molecular imaging, biomarkers, and biosensors. The human eye is a complex organ that is separated from the rest of the body by number of layers of biological barriers. However, the internal ocular structures and tissues are protected from the external environment by the tight junctions of the corneal epithelium and the mucosal surface $[1,2]$.

The major challenge faced by today's pharmacologist and formulation scientist is ocular drug delivery. The ophthalmic application of drugs is the primary route of administration for the treatment of various eye diseases, and is well-accepted by patients; usually, only small amount of the drug administered penetrate the cornea to reach the desired intraocular tissue due to corneal barriers and dilution caused by lacrimation [3]. Delivery of drugs to the targeted ocular tissues is restricted by various precorneal, dynamic and static ocular barriers [4]. Hence, frequent instillation of concentrated solutions is needed to obtain the desired therapeutic effect in both the anterior and posterior hemispheres of the eye. But this can cause corneal damage and undesirable side effects resulting from the systemic absorption of drugs through the nasolacrimal duct [5,6]. Therefore, the main challenge for ocular drug delivery is how to deceive these protective barriers in order to achieve therapeutically effective concentrations of drugs in the intraocular tissues.

In the past two decades, ocular drug delivery research acceleratedly advanced towards developing a novel, safe and patient compliant formulation and drug delivery devices/ techniques, which may surpass these barriers and maintain drug levels in tissues. In this regard, it is important to increase the effectiveness of drugs by enhancing their bioavailability [7]. In order to increase drug bioavailability and overcome these problems, several strategies like development of conventional topical formulations such as suspensions, emulsions and ointments; preparation of viscous solutions, micro/ nanoparticles, modulation of conventional topical solutions with permeation and viscosity enhancers and hydrogels have been developed and investigated [1,4, 8-11].

Various nano formulations have also been introduced for anterior segment of ocular delivery. On the other hand, for posterior ocular delivery, research has been immensely focused towards development of drug releasing devices and 
nanoformulations for treating chronic vitreo-retinal diseases. These novel devices and/or formulations may help to surpass ocular barriers and associated side effects with conventional topical drops. Also, these novel devices and/or formulations are easy to formulate, no/negligibly irritating, possess high precorneal residence time, sustain the drug release, and enhance ocular bioavailability of therapeutics [12].

Ophthalmic formulations containing nanoparticle drug show a better solution to the limitations surrounding ocular drug penetration $[9,10]$, and it is known that to circumvent the side effects related to drug delivery, decreasing direct cellular stimulation and reducing the amount drug used by increasing its bioavailability are useful ways [7]. It is expected that ophthalmic drug systems using nanoparticles may provide an alternative way for increasing ocular drug penetration $[9,10]$.

Nanosuspensionsor suspensions in general are heterogeneous systems, their stability and therapeutic efficacy is significantly dependent on the physicochemical characteristics of the system. Within the confines of these features, the physicochemical properties of the dispersed phase (suspended solids) are even more critical in deter $\neg$ mining the stability of nanoparticulate systems [13]. These features include, but are not limited to, solubility, particle size, crystal habits of the suspended solids, and the interactions resulting from some of these factors. As nanosuspension systems are essentially colloidal in nature, a number of features defining colloidal systems can be employed to elucidate the physicochemical characteristics of nanosuspension [12].

\section{Considerations of Nanosuspension for Ocular Delivery}

The particle size influences its functionality in terms of its uptake, residence in circulation, adherence, degradation, clearance. The particles' fate inside the body has been reported as: $\geq 2 \mu \mathrm{m}$, trapped inside liver cells; $\geq 300-400 \mathrm{~nm}$, captured by macrophages and excreted; $\geq 200 \mathrm{~nm}$, filtered in the spleen; $\geq 100 \mathrm{~nm}$, escape from blood vessels through the endothelial lining. Thus, the movement of nanoparticles inside tissues is governed by size. In the ophthalmic field, nanosuspension of size range 10 to $1000 \mathrm{~nm}$ allow for the improved topical passage of large, water insoluble molecules through the barriers of the ocular system [14]. Superficial barriers delay direct and systemic drug access to the specific site of action. Drug-loaded nanosuspension show prolonged residence time for eye drops, increased ability of the drug to penetrate into the deeper layers of the ocular structure and aqueous humor thus minimizing precorneal drug loss caused by rapid tear fluid turnover and decreased toxicity $[7,15]$.

An ideal ocular drug delivery system should possess key properties that include:

A. Controlled and sustained release profile to maintain the therapeutic concentration of the drug over a prolonged period of time to reduce the frequency of administration;
B. Specific targeting and prolonged retention in the diseased tissues to improve therapeutic efficiency and avoid side effects;

C. Patient-friendly delivery routes that eliminate or minimize

\section{Ocular Barriers}

The unique structural and functional properties of the eye are synchronized by visual cells and transparent tissues. The regulatory mechanism of this organ relies mainly on tight cellular barrier between eye's anterior and posterior segments which controls fluids and solutes traverse through membranes (Figure 1). Similarly, drug transport via these barriers is also highly controlled and limited; hence, application of novel drug delivery/ targeting strategies for effective pharmacotherapy seems to be crucial. Recent advances in novel nano-sized delivery targeting/delivery agents are generating new insights for the ocular disease therapy [13]. However, efficient delivery and adequate bioavailability of such medications should be verified $[16,17]$.

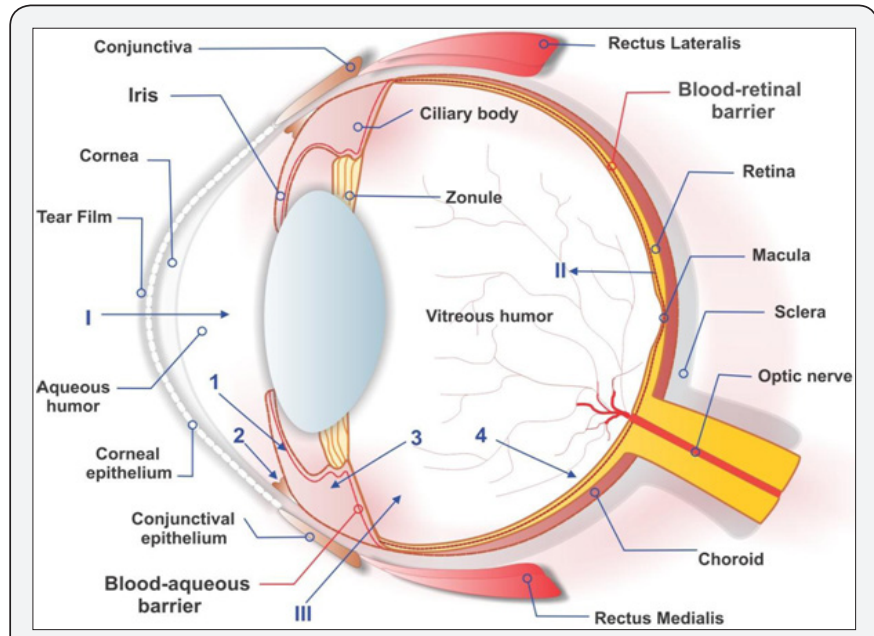

Figure 1: Schematic illustration of the main structure of the eye and the ocular barriers.

\section{Blood ocular barriers}

Normal ocular structure and visual function properties are maintained by blood ocular barriers consisting of two main components (i.e. blood aqueous barrier (BAB) and blood retinal barrier (BRB)). This barrier physically separates blood vessels from internal segment of eye, controlling passage of any particle/chemical into ocular tissues. As illustrated in Figure 1, ocular medications administered via local or systemic routes must overcome this barrier to achieve adequate concentration and maintenance in retina and vitreous. Furthermore, blood ocular barrier maintains tissue/fluid composition and produces aqueous humor (Cunha-Vaz et al. 1997a). The control of inflow/ outflow of aqueous humor provides the sufficient pressure inside the eye [18]. 


\section{Blood-aqueous barrier}

The BAB is formed by tight junctions of iris vascular endothelium and non-pigmented layer of ciliary epithelial cells (Figure 1). This structure is located in the anterior part of the eye, preventing undesired traverse of exogenous materials into the ocular posterior segment and providing transparency and chemical equilibrium of the ocular fluids Cunha-Vaz et al. [19]. It is of note that iris blood vessels withstand macromolecule passage, whilst capillary of ciliary body is less restrictive and allows favored outward traverse of substances toward systemic circulation. Moreover passive transport through BAB, results in rapid elimination of substances with small molecular size and more lipophilicity as compared to large and hydrophilic molecules. For example, the clearance of pilocarpine is $13.0 \mu \mathrm{l} / \mathrm{ml}$ in rabbits whereas inulin clearance is close to the rate of aqueous humor turnover (i.e. $3.0-4.7 \mathrm{ml} / \mathrm{min}$ ) [20].

\section{The blood-retinal barrier}

The blood-retinal barrier (BRB), which locates in the posterior part of the eye, contains two types of cells, i.e. 1) the retinal capillary endothelial (RCE) cells, and 2) the retinal pigment epithelial (RPE) cells. These cells from the inner and outer BRB, respectively. Basically, the specialized transport processes and the restrictive barrier functions RPE selectively control the transportation of nutrients/compounds, by which functionalities only designated nutrients can be traversed between choroid and retina [21]. The inner BRB covers the lumen of retinal capillaries that are able to selectively protect the retina from circulating molecules of the blood. Given these facts, it appears that the satisfactorily delivery and efficient pharmacological effects of drugs within the vitreous and the retina require systemic or intravitreal drug administration. Nevertheless, systemic application via oral or intravenous administration necessitates very high doses of the drug because the blood flow and restrictive functionality of BRB allow only very trivial fraction of the drug to reach the posterior chamber (typically only 1-2\% of the concentration in the plasma). But, it should be evoked that administration of large portion of the drug can be associated with inadvertent adverse consequences [22].

\section{Strategies to Overcome Blood Ocular Barriers}

In recent years, for treatment of many ocular disorders, there has been a profound shift towards implementation of more efficient treatment paradigms. For example, the neurodegenerative disorder "glaucoma" which is associated with elevated intraocular pressure has affected many patients' lives, while its treatment has fortunately moved from the management of intraocular pressure to the prevention of neuro degeneration and maintenance of retinal function [23]. The artificial tears is no longer the main treatment for the dry eye which is a common cause of patient visits to eye care specialists damaging the ocular surface. It is now being controlled with Restasis ${ }^{\circledR}$ (cyclosporin A ophthalmic emulsion), which targeted the immune component of the disease [14].
The primary physiologic blockage against installed drugs is the tear film. Cornea is the main route for drug transport to the anterior chamber (I). The retinal pigment epithelium and the retinal capillary endothelium are the main barriers for systemically administered drugs (II). Intravitreal injection is an invasive strategy to reach the vitreous (III). The administered drugs can be carried from the anterior chamber away either by venous blood flow after diffusing across the iris surface (1) or by the aqueous humor outflow (2). Drugs can be removed from the vitreous away through diffusion into the anterior chamber (3), or by the blood-retinal barrier (4) [16].

\section{Ocular Drug-Delivery Mechanisms}

To enter the cellular interior, any compound needs to cross biological mem $\neg$ branes and barriers. Cell membrane imposes a barrier functionality to restrict the free traverse of particles/ solutes via lipid bilayer (Payghan et al. 2017). However, the necessary compounds are shuttled into target site via passive/ active path $\neg$ ways by employing various transport machineries, that is, carrier-mediated or endocytosis/transcytosis mechanisms.

\section{Simple passive transport}

Passive transport across the virtually all cell membranes is nonspecific and requires no energy. Molecules such as dissolved gases (e.g., $\mathrm{O}_{2}$ and $\mathrm{CO}_{2}$ ), small uncharged molecules and hydrophobic substances traverse the phospholipid bilayer in a direct proportion of their concentration gradient. Furthermore, lipid solubility and partitionation in the membranous lipid bed is a key factor in determination of the diffusion extent and rate of hydro $\neg$ phobic molecules. On the other hand, facilitated transport (carrier-mediated diffusion) is employed for the transport of vital ions or glucose in their con $\neg$ centration gradient direction in a higher rate compared to simple passive dif $\neg$ fusion [24].

\section{Carrier-mediated transport}

There exist a number of uni/bidirectional influx/efflux transporters that assist in the essential nutrient supply of the cells such as glucose transporters, amino acid transporters, peptide transporters, and monocarboxylate transporters [15]. The glucose transporter expression and functionality have been exten-sively studied; and confirmed the widespread localiza $\neg$ tion of this transporter in ciliary body, iris, retinal and in some species in the trabecular meshwork and lens [25]. Distribution and role of cationic amino acid transporters (CATs) in ocular tissue have been studied [26]; CAT1 and CAT2 are expressed in ocular surface as well as lacrimal apparatus that are differently regulated in various pathophysiologi $\neg$ cal conditions. Ocular membrane transporters possess a main challenge for xenobiotic delivery and targeting of the eye and have been discussed broadly.

\section{Ocular transport machineries}

Cell membranes impose a barrier to the free movement of molecules through a membranous lipid bilayer and associated 
transport machineries. A solᄀute, based upon molecular properties, is transported across the cell mem $\neg$ branes by passive diffusion and/or carrier/receptor-mediated transport [15]. Nearly most of the ocular tissue displays $\mathrm{Na}+\mathrm{H}+$ exchanger, $\mathrm{Na} /+-\mathrm{HCO} 3$ symporter, and $\mathrm{Cl} /--\mathrm{HCO} 3$ exchanger that are involved in the regulation of intracellular $\mathrm{pH}$. The $\mathrm{Na}+\mathrm{H}+$ exchanger is present at the basolateral membranes of both epithelial and endothelial cells, while $\mathrm{Na} /+\mathrm{HCO} 3$-transporter is predominantly localized at the basolateral side of the corneal endothelium and is faintly expressed in the corneal epithelium [27]. This implies that the distribution pattern of these transporters at the apical and basolateral membrane varies depending upon the cellular needs and physiologic functions. The influx and efflux transport machineries are functionally expressed in the main membranous barriers of the eye, that is, the cornea; the conjunctiva, the iris-ciliary body, and the retina. The unidirectional/bidiᄀrectional influx transporters such as monocarboxylate transporters (MCTs), glucose transporters (e.g., Glut1), amino acid transporters (LAT1 and LAT2), and PepT1 supply essential nutrient requirements of the cells. The LAT1 transporter of the brain capillary endothelial cells functions bidirection $\neg$ ally with greater efflux activity [15] (Figure 2).

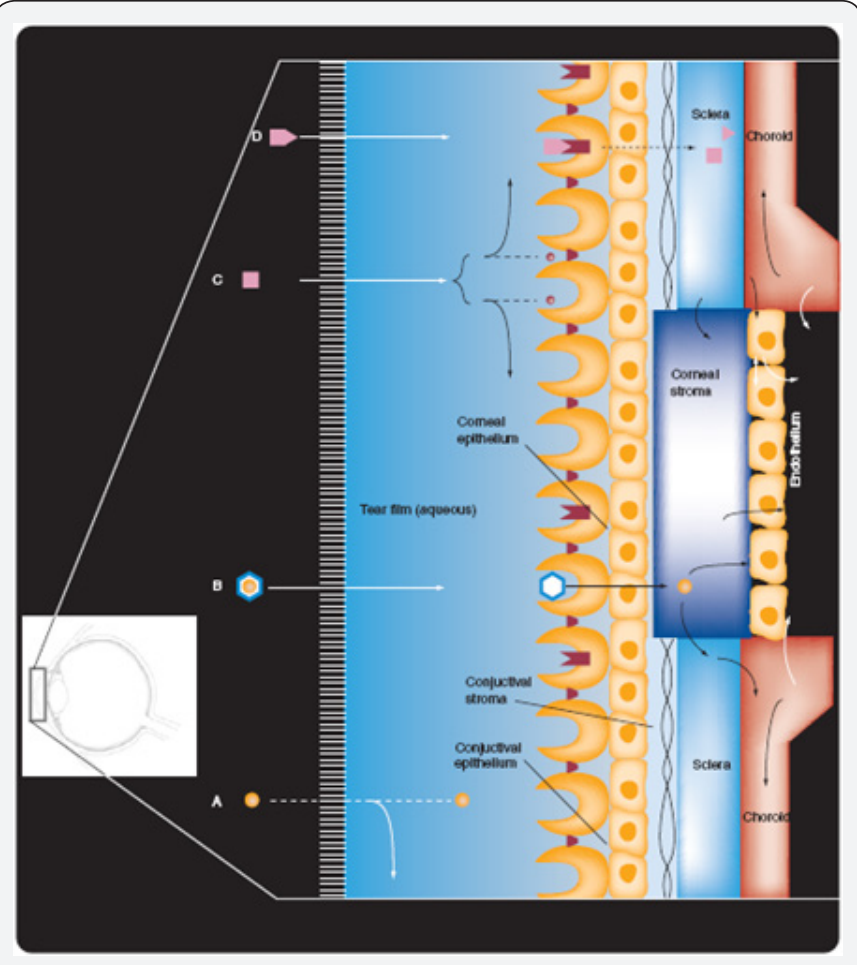

Figure 2 : Transport of drugs through the ocular surface. (Maryam et al. 2008)

a) A lipophilic drug that cannot easily penetrate the tear film is washed away.

b) A lipophilic drug in the central cavity of a cyclodextrin molecule. The cyclodextrin solubilizes in the tear film and reaches the ocular epithelium. The lipophilic drug partitions out of the cyclodextrin and into the lipid membrane of the epithelium.

c) A hydrophilic drug that solubilizes in the tear film and reaches the epithelium; it cannot cross the epithelium transcellularly (because of the lipid membrane) or paracellularly (because of tight junctions) and eventually washes away from the eye surface.

d) A hydrophilic prodrug that penetrates the epithelium transcellularly with the aid of a membrane transporter. Once in the ocular tissue, it is converted into the drug by enzymes. The corneal and conjunctival epithelia are contiguous and contain several layers of cells (not shown), the outermost layer features microvilli that interact with tear film mucins. Drugs that penetrate the epithelia can move easily between ocular tissues, such as the corneal and conjunctivalstroma, the sclera beneath the conjunctiva, the vascularized choroid and the leaky endothelium. From there, they can diffuse into the anterior chamber or laterally through the sclera to the eye posterior.

\section{Endocytosis and transcytosis}

Receptors are mainly involved in endocytosis or transcytosis of compounds, which contribute to some degree of specificity or selectivity. Clatrin-mediated vesicular uptake is more scrutinized and accounts for most of the receptor-mediated delivery, though caveolae route or other unidentified pathways are not negligible [24].

\section{Topical absorption of ocular drug}

Corneal and/or noncorneal routes are the main approaches for the local drug therapy of the eye. However, it should be noted that in the eye cul-de-sac, the medications are subjected to some physiological/biological barriers. First, the administered pharmaceuticals may be carried away by the lacrimal fluids. Second, inevitable systemic absorption occurs through the conjunctival sac as well as the nasal cavity. The corneal route represents the main absorption path for most of the ophthalmic therapeutics. However, corneal absorption is also considered to be a rate-limiting process due to the presence of the cor $\neg$ neal epithelium. The second path involves penetration across the conjunctiva and sclera into the intraocular tissues; however, this path appears to be less productive due to the presence of the local capillary beds that remove the drug from target sites to the general circulation [24].

\section{Ocular Pharmacotherapy}

The mechanisms such as reflex blinking, lacrimation, tear turnover, and drainage impose absorption problem to the topically applied ophthalmic drugs, resulting in poor absorption of the administered pharmaceuticals and rapid removal of foreign substances from the eye surface. Thus, to main 7 tain a therapeutic drug level in the tear film or at the site of action, repeated instillations of eye drops are expected. However, such frequent use of medi $\neg$ cations can induce inadvertent consequences due 
to exposure of highly con $\neg$ centrated drug solutions to the ocular tissues, hence resulting in undesired side effects at the ocular surface. While conventional pharmaceuticals are often associated with such predictable bio impacts, advanced pharmaceu-ticals may provide better corollaries with high patient's compliance. Thus, here, we intend to provide an overview on the conventional and advanced pharmaceuticals used for eye diseases [24].

\section{Conventional Pharmacotherapy of Ocular Diseases}

Conventional pharmaceuticals (e.g., eye drops, gels, creams, and ointments) are widely used for the treatment of some eye diseases. In general, the lower is the solubility of a drug in the vehicle, the higher is the chemical potential and the more readily is the drug partitioning into the release medium. Thus, various ocular pharmaceuticals (viscous gel, cream, or ointment) have been designed to release the drugs basically through diffusion and partitioning. Delivery of macromolecules can be more sustained in hydrogels compare to small drug molecule such as pilocarpine and the design of sustained-release dosage forms is largely dependent upon physicochemical properties of drugs and biological site of application. The ophthalmic formulations usually conᄀtain high concentrations of their active ingredients because of the relatively poor penetration of the topical use. Therefore, the conventional dosage forms may elicit toxic effects in ocular cells/tissues [24].

It has been reported that the topical pharmacotherapy of the eye may be significantly influenced by some factors such as the drainage of the instilled solution, lacrimation and tear turnover, metabolism, tear evaporation, non $\urcorner$ productive absorption, limited corneal area and poor corneal permeability, and binding by lacrimal proteins [28]. Therefore, sufficient corneal penetration and prolonged contact time with the corneal tissue is of essence for effective and successful ocular drug absorption. To increase drug absorption in the eye, various modalities have been investigated, including: sustained-release formulations such as biodegradable implants [29], device DDS (e.g., collagen shields, iontophoresis, and pumps iontopho $\neg$ resis) [30], prodrugs such as acyclovir dipeptide esters [28], ion pair for $\neg$ mation [31], bioadhesives, and mucoadhesives [32].

In addition to topical pharmacotherapy, ocular diseases in posterior segments (retinal pathologies such as fungal and bacterial endophthalmi $\neg$ tis, viral retinitis, and proliferative vitreal retinopathies) require systemic administration. Nevertheless, systemic pharmacotherapy of the eye is often attributed with limited success mainly because of the excellent $\mathrm{BRB}$ restric $\neg$ tiveness excluding the organ from systemic circulation [16] and selectively control the inward and outward travers by transport machiner $\neg$ ies [24].

a) For noninvasive intraocular delivery, some major considerations should be taken into account, including: safe passage of a drug across the cornea to reach the site of the action b) prolong duration of drug action within the target site to minimize the frequency of drug administration

c) specific tar $\neg$ geting of the desired biomarkers to localize the desired pharmacodynamics action with minimal impacts on nonspecific tissues

d) Effective evading from BAB.

While most of the currently used medications for the treatment of the ocular diseases (e.g., glaucoma, conjunctivitis, keratitis, and uveitis) were shown to have bioavailability problems [28], novel advanced technologies are deemed to favor ocular pharmacotherapy. The ocular DDS have so far been improved by development of novel pharmaceuticals [8]. Of these, multifunctional multimodal nano $\neg$ medicines appear to be the mainstay platform for advanced medicines. Thus, we will discuss this approach in more detail. (Barar et al. 2006)

\section{Applications of Nanosuspensions in Ocular Delivery}

\section{Ocular inflammation}

Nanosuspensions can be defined as sub-micron colloidal system which consists of poorly water-soluble drug, suspended in an appropriate dispersion medium stabilized by surfactants. Nanosuspensions usually consist of colloidal carriers like polymeric resins which are inert in nature. They help in enhancement of drug solubility and thus bioavailability. Unlike microemulsions, they are also popular because of their non irritant nature.

The steroidal and non-steroidal anti-inflammatory drugs (NSAIDs) are routinely used in ocular surgeries, even though they often impose some adverse reactions. These medications are the most studied drugs to be exploited as ocular nanomedicines $[33,34]$. Accordingly, localized therapy of ocular inflammation by these pharmaceuticals need to be optimized since most ocular diseases are classically treated with topical eye-drops which usually require frequent utilization of highly concentrated solutions. Enormous efforts, thus, have so far been devoted to maximize the localized delivery and targeting of desired pharmaceuticals using hydrogels, micro- and/or nanoparticles and liposomal formulations [35].

Adibkia et al. [35] reported that nanosuspension of piroxicam can control the endotoxin-induced uveitis (EU) in rabbits Adibkia et al. [36], where cationic polymer (i.e. Eudragit @ RS100) was used to formulate nanosuspensions of piroxicam by means of solvent evaporation/extraction technique (also called single emulsion technique). Similar studies were carried out using piroxicam in Eudragit RS 100. In vivo studies in rabbits have shown significant anti-inflammatory effects compared to micro suspensions Adibkia et al. [35]. Given the cellular responses to the lip polysaccharide (component of gram-negative bacteria cell wall) induced uveitis Koizumi et al. [37], it can be assumed that the piroxicam nanosuspensions perhaps favor the cellular recovery from EU by conferring a better therapeutic effect 
because of increased cellular uptake and enhanced inhibitory mechanism on the expression of the inflammatory mediators.

Adibkia et al. [35] reported that the Eudragit ${ }^{\circledR}$ RS100 possesses an appropriate stability and size distribution characteristics together with its positive surface charge of about $30 \mathrm{mV}$, it is considered as a suitable ocular DDS Pignatello et al. [38]. The positively charged nanoformulations may interact with anionic mucins presented in the tear film, and cause consequential prolongation of drug residency time on the corneal surface Dillen et al. [39]. Besides, the nanosuspensions may also confer more comfortableness for and better acceptance by patients in comparison with the routine ophthalmic suspensions that are basically formulated in micrometer ranges and show poor characteristics Zimmer et al. [34].

\section{Controlled release ocular delivery}

Developing successful drug delivery systems for releasing active molecules to the eye anterior or posterior segments is an exciting challenge for modern pharmaceutical technology. For drugs showing problems of solubility or stability in the eye fluids, or difficulty passing through the corneal barrier, a controlled-release system can help to modulate and/or sustain the amount of drug released, reducing the drainage from the eye surface and the unproductive absorption usually associated with conventional aqueous eye drop formulations [40], Hamsika et al., 2006; Ripal et al., 2009; Nakhlband et al., 2011).

Use of polymeric nanoparticles is one of the most interesting approaches to achieving local controlled drug delivery Wood et al. 1986; [41-43]. In this field, Eudragit Retard polymer nanoparticle suspensions have been investigated as a carrier system for the ophthalmic release of non steroid alanti inflammatory drugs, such as ibuprofen and flurbiprofen Bucolo et al. [38]; Pignatello et al., 2002b; Pignatello et al. [44]. Such carriers showed an interesting size distribution and a positive surface charge, along with good stability, that make them potential candidates for use in ocular drug delivery systems. In particular, their positive surface charge ( $\zeta$-potential) can allow a longer residence time of nanoparticles on the cornea surface, with a consequent slower drug release and higher drug concentrations in the aqueous humor, compared with classical eye drops.

Eudragit RS100 (RS) and RL100 (RL) polymers are being used for the enteric coating of tablets and for preparing controlled-release formulations. RS and RL are copolymers of poly (ethylacrylate, methyl-methacrylate and chlorotrimethylammonioethyl methacrylate), containing an amount of quaternary ammonium groups ranging between $4.5 \%$ and $6.8 \%$, and $8.8 \%$ and $12 \%$, for RS and RL, respectively. These matrixes are insoluble in water at physiological $\mathrm{pH}$ values and capable of swelling, so they are good for the dispersion of active compounds (Kawashima et al., 1993; Perumal et al. [45]; Pignatello et al. [46].

Similarly, nanosuspensions of methylprednisolone acetate (MPA) using ERS to pursue their impacts on the inhibition of inflammatory symptoms in rabbits with EU. It was found that the utilization of MPA-ERS nanosuspensions confers a controlled ocular delivery of MPA Adibkiaet al. [35]. Although molecular biology aspects of such therapies for uveitis is yet to be mechanistically investigated, it appears that the application of these types of nanosuspensions as a non-invasive approach seems to be safer controlled ocular delivery of anti-inflammation agents for inhibition of the uveitis symptoms. Similar results have been reported for flurbiprofen and ibuprofen Pignatello et al. [38]; Pignatello et al. 2002b.

\section{Sustained release ocular delivery}

Nanosuspensions can prove to be a boon for drugs that exhibit poor solubility in lachrymal fluids. For delivery of such drugs, approaches such as suspensions and ointments have been recommended. Although suspensions offer advantages such as prolonged residence time in a cul-desac (which is desirable for most ocular diseases for effective treatment) and avoidance of the high tonicity created by water-soluble drugs, their actual performance depends on the intrinsic solubility of the drug in lachrymal fluids. Thus, the intrinsic dissolution rate of the drug in lachrymal fluid governs its release and ocular bioavailability. However, the intrinsic dissolution rate of the drug will vary because of the constant inflow and outflow of lachrymal fluids. Hence, suspensions may fail to give consistent performance. However, nanosuspensions, by their inherent ability to improve the saturation solubility of the drug, represent an ideal approach for ocular delivery of hydrophobic drugs (Patel et al., 2013).

Moreover, the nanoparticulate nature of the drug allows its prolonged residence in the cul-de-sac, giving sustained release of the drug. To achieve sustained release of the drug for a stipulated time period, nanosuspensions can be incorporated in a suitable hydrogel base or mucoadhesive base or even in ocular inserts Pignatello et al. [38]; Pignatello et al. 2002b. An approach that has recently been investigated to achieve the desired duration of action of the drug is the formulation of polymeric nanosuspensions loaded with the drug. The bioerodible as well as water soluble/ permeable polymers possessing ocular tolerability Pignatello et al. [38]; Pignatello et al. $2002 \mathrm{~b}$ could be used to sustain the release of the medication.

These nanosuspensions also produce sustained drug release and were more effective over a longer duration. Nanosuspensions also impart stability to the drug in the formulation. Cloricromene was formulated in nanosuspensions by using Eudragit RS100 and RL100. AD6-loaded Eudragitretarded nanoparticle suspension offered a significant edge in enhancing the shelf life and bioavailability of the drug following ophthalmic application Pignatello et al. [40]. The ERL nanoparticles containing Cloricromene (8-monochloro-3-b-diethylamino ethyl-4-methyl7-ethoxy-carbonyl-methoxycoumarin HCL; AD6) synthetic coumarin derivative that possesses antithrombotic and anti platelet actions, inhibits polymorpho nuclear cells (PMN) neutrophil function, and causes vasodilatation (Squadrito et al. 1991; Galli et al. 1980; Porcellati et al. 1990; Travagli et al. 1989) with positive zeta potential values $(+27.3 \mathrm{mV})$ and a particle size 
of $80 \mathrm{~nm}$ were topically applied to rabbit eyes and showed no sign of toxicity or irritation to ocular tissues. A sustained release was observed in vitro as well as in vivo, resulting in a doubled AUC compared with an aqueous solution (Bucolo et al. 2004).

Overall, all nanoparticles showed a prolonged release profile without burst effect, in which the complete release of drug after $24 \mathrm{hr}$ (obeyed from Higuchi diffusion controlled model kinetics) explicitly indicate that there exists a structural homogeneity of the polymeric matrix, and also a more uniform distribution of the drug (Bucolo et al. 2004). Given that the ester form AD6 is freely soluble but unstable in aqueous solutions, where it is rapidly converted into the insoluble acid form, a delivery system able to ensure a slow and prolonged release of this active form at an intraocular level could improve the therapeutic benefits of this pharmacological agent. Therefore, in present work the feasibility of RS and RL matrices for the preparation of AD6loaded ophthalmic drug delivery systems was investigated (Porcellati et al., 1990; Travagli et al. 1989).

Modeling of drug release from nanoparticles of ciprofloxacin: Eudragit ${ }^{\circledR}$ has also been described by Dillen [39], whose work showed that the release rate data fitted to the Higuchi's kinetic model. Based on our findings, treatment with piroxicam nanosuspensions significantly reduced observational symptoms of uveitis (Bucolo et al. 2004) (based on Hogan's classification method) such as redness, presence of fibrin, photophobia, and lacrimation (Bu et al. 2007; Koizumi et al. [37]. We may assume that the prolonged impacts of piroxicam nanosuspensions may be due to its interaction with local cellular components because of the positive surface charge of the nanoparticles in addition to the greater penetration and cellular uptake (Pignatello et al. [38]; Pignatello et al. 2002b).

\section{Topical Release of NSAID's In Ocular Delivery}

Topical application of non-steroidal anti-inflammatory drugs on the eye is a common treatment used to contrast the miosis induced by surgical traumas, such as cataract extraction (Patravale et al. 2004). With the aim of improving the availability of sodium ibuprofen (IBU) at the intraocular level, IBU-loaded polymeric nanoparticle suspensions were made from inert polymer resins (Eudragit RS100) (Pignatello et al. [44].

The nanosuspensions were prepared by a modification of the quasi-emulsion solvent diffusion technique using variable formulation parameters (drug-to polymer ratio, total drug and polymer amount, stirring speed). Nanosuspensions had mean sizes around $100 \mathrm{~nm}$ and a positive charge (z-potential of $140 / 160 \mathrm{mV}$ ), this makes them suitable for ophthalmic applications. Stability tests (up to 24 months storage at $48{ }^{\circ} \mathrm{C}$ or at room temperature) or freeze-drying were carried out to optimize a suitable pharmaceutical preparation. In vivo efficacy was assessed on the rabbit eye after induction of an ocular trauma (paracentesis). An inhibition of the miotic response to the surgical trauma was achieved, comparable to a control aqueous eye-drop formulation, even though a lower concentration of free drug in the conjunctival sac was reached from the nanoparticle system. Drug levels in the aqueous humour were also higher after application of the nanosuspensions; moreover, IBU-loaded nanosuspensions did not show toxicity on ocular tissues [48-50].

The inflammatory response of the ocular tissues is a common side-effect associated to ophthalmic surgery. It can interfere with the normal function of the eye, whose optic transparency must be maintained. Together with steroidal agents, nonsteroidal anti-inflammatory drugs (NSAIDs) are used in the eye surgery. They can however induce burn and worse the flogistic process, by blocking prostaglandin (PGs) synthesis through the inhibition of cyclooxygenase, and improving the production of other inflammation mediators, such as leukotrienes (Masuda et al. 1984) [51-55].

Surgical or mechanical traumas of the anterior segment of the eye induce a vascular inflammatory reaction due to the disruption of the blood-aqueous barrier, with a marked rise in protein content of the aqueous humour, a transient ocular hypertension and miosis (Kulkarni et al. 1991). These considerations are important since miosis is a frequent problem during extra capsular cataract surgery, despite the instillation of topical mydriatic agents (Sabiston et al. 1987; Gimbel et al. 1989; Brown et al., 1996) [56-60].

Arylpropionic-type NSAI drugs, like flurbiprofen (FLU) can antagonize the pupillary constriction during the intra-ocular surgery, by inhibiting the cyclooxygenase. The prevention of inflammation mediator release in the anterior eye segment can also decrease post operatory time after intra-ocular surgery. Among available NSAI agents, FLU and diclofenac are the most used ones (Brown et al., 1996; Keates et al. 1984; Kocak et al. 1998; Thaller et al. 2000). Studies on the rabbits showed that FLU and phenylefrine maintain the mydriasis induced by atropine after paracentesis (an evacuating corneal injection which starts the inflammatory process) (Bucolo et al. 1995). NSAIDs block the cyclooxygenase pathway and reduce polymorpho-nuclear leukocytes (PMNs) infiltration in the aqueous humour through a mechanism that does not involve leukotriene activity, and decreases significantly the postsurgical oedema after intraocular surgery. The clinical interest in the reduced PMNs concentration is important since these compounds participate in the events associated with the initiation and continuation of ocular inflammation, in the process that leads to corneal damage (Kenyon et al., 1987; Csukas et al., 1990).

Flurbiprofen encapsulated in Eudragit RS $100 \AA$ and RL $100 \AA$ polymer resins prevents myosis, which might be induced during extra capsular cataract surgery (Pignatello et al. 2002b). Charge on the surface of nanoparticles facilitates its adhesion to the cornea. Animal studies have revealed that anti-inflammatory effect of nanosuspensions was more than micro suspensions.

\section{Ocular bioavailability of nanosuspension}

Poorly soluble drugs are very often a challenging problem in drug formulation, especially when the drugs are poorly soluble 
simultaneously in aqueous and non-aqueous media (Patel et al., 2013). This leads in most cases to poor bioavailability or poor erratic absorption of these drugs (Merisko-Liversidge et al., 2003; M"uller et al. [47]. Many attempts have been made to increase the saturation solubility of poorly soluble drugs (Liversidge et al., 1992; Sucker, 1998). Recently, drug micro-particle suspensions can be milled by applying a high pressure homogenization process M"uller et al. [47]; Jacobs et al., 2001; Keck and M"uller 2006) leading to a product called nanosuspension. Nanosuspensions are colloidal dispersion of submicron drug particles stabilized by polymer(s) or surfactant(s). It is emerged as promising strategy for delivery of hydrophobic drugs. For ocular delivery, it provides several advantages such as sterilization, ease of eye drop formulation, less irritation, increase precorneal residence time and enhancement in ocular bioavailability of drugs which are insoluble in tear fluid (Patravale et al., 2004; M"oschwitzer et al., 2004).

An outstanding feature of the nanosuspension is the increase in saturation solubility and consequently an increase in the dissolution rate of the compound [47]; M"uller et al., 2001; Merisko-Liversidge et al., 2003; Rabinow, 2004). Ophthalmic drug delivery, more than any other route of administration, may benefit to a full extent from the characteristics of nanosized drug particles. The nano-size represents a state of matter characterized by higher solubility (Keck et al., 2006; Rabinow et al., 2004) higher surface area available for dissolution (Bisrat and Nystr"om, 1988; Mosharraf and Nystr"om, 1995), higher dissolution rate (Zhang et al., 2006), higher bioadhesion (Duch^ene and Ponchel, 1997; Yoncheva et al., 2005) and corneal penetration.

It has been recommended that particles be less than $10 \mathrm{~nm}$ to minimize particle irritation to the eye, decrease tearing and drainage of instilled dose and therefore increase the efficacy of an ocular treatment. Many published articles have indicated the importance of particle size in ophthalmic bioavailability (Hui and Robinson et al. 1986; Schoenwald and Stewart, et al. 1980); most of these articles prove that decreasing the particle size increases the ophthalmic bioavailability. On the other hand, the use of nanosuspensions for improving the ophthalmic bioavailability has been discussed (Pignatello et al., 2002 a,b).

The efficacy of nanosuspensions in improving ocular bioavailability of three different types of glucocorticoids; hydrocortisone, prednisolone and dexamethasone which are practically water insoluble has been demonstrated in several research studies (Kassem et al., 2007; Ali et al. [9]; Armaly et al., 1986). They represent the three classes of short, medium and long acting steroids, respectively. They are widely used topically for the treatment of inflammatory conditions of the conjunctiva and anterior segment of the eye. The present therapy with these drugs, mostly dictates frequent instillation in the conjunctival sac, which, besides leading to poor patient compliance, may result in administration of a large dose which, in turn, may induce glaucoma, cataract formation and damaged optic nerve
(Armaly, 1986). The present study addresses hydrocortisone, prednisolone and dexamethasone suspensions in the submicron range (nanorange) in the form of nanosuspensions using high pressure homogenizer. The nano size range represents a state of matter different from the conventional micro-crystalline suspensions so far used in practice in order to optimize their biological performance.

For instance, (Kassem, et al. 2007) compared ocular bioavailability of various glucocorticoids (prednisolone, dexamethasone and hydrocortisone) from nanosuspensions, solutions and microcrystalline suspensions. The formulations were instilled into the lower culde-sac of the rabbit eye and intraocular pressure (IOP) was measured at frequent time intervals up to $12 \mathrm{~h}$. The area under percentage increase in IOP vstime curve (AUC) values for all the suspensions were higher than that for the respective drug solutions. In addition, higher extent of drug absorption and more intense drug effects were observed for all steroids form nanosuspensions compared with solutions. In another study, Ali et al [9] compared ocular bioavailability of hydrocortisone (Hc) nanosuspensions prepared by precipitation and milling method with HC solution in rabbits post topical instillation. A sustained drug action which was represented in terms of changes in intraocular pressure was maintained up to $9 \mathrm{~h}$ for the nanosuspensions compared to $5 \mathrm{~h}$ for the drug solution. It can be concluded that nanosuspensions could be an efficient ophthalmic drug delivery system for delivery of poorly soluble drugs. In addition, nanosuspension can also be incorporated into hydrogels or ocular inserts for achieving sustained drug release for stipulated time period (Patel et al., 2013).

\section{Conclusion}

Nanosuspension formulation have been largely solved the solubility as well as dissolution problems to improve drug absorption. Attractive characteristics of nanosuspensions such as uniform nano sized particles, improved solubility in biological media and adhesiveness (sugar versus ground sugar), increased drug concentrations, and residence time at the absorption sites enable the innovative design of a new class of drug delivery systems. Such nano sized drug formulations have a number of benefits for drug therapies including high surface area, controllable nano size dimensions, and modified surface chemistry. A nanosuspension formulation solves the poor solubility problems, but also improves drug efficacy. The aim of future studies is to combat the challenges associated with poorly soluble drugs in order to achieve high bioavailability, dissolution velocity, and bioadhesion of the drug. The applications of nanosuspensions in oral and parental routes have been very well established, although applications in pulmonary and ocular delivery have to be evaluated.

\section{References}

1. Diebold, Y, Jarrín M, Sáez, V, Carvalho EL, Orea M, et al. (2007) Ocular drug delivery by liposome-chitosan nanoparticle complexes (LCS-NP). Biomaterial 28(8): 1553-1564. 
2. Baba K, Tanaka Y, Kubota A, Kasai, H, Yokokura SA, et al. (2011) method for enhancing the ocular penetration of eye drops using nanoparticles of hydrolyzable dye. J Controlled Release 153(3): 278-287.

3. Li X, Zhang Z, Li J, Sun S, Weng Y, et al. (2012) Diclofenac/biodegradable polymer micelles for ocular applications. Nanoscale 4(15): 4667-4673.

4. Bhattacharjee H (2012) Nanosuspensions in Ocular Drug Delivery Systems. In: Thassu D, Gerald J, Chader [Eds.]. Ocular Drug Delivery Systems: Barriers and Application of Nanoparticulate Systems, CRC Press, USA, pp. 401-432.

5. Asasutjarit R, Thanasanchokpibull S, Fuongfuchat A, Veeranondha S (2011) Optimization and evaluation of thermo responsive diclofenac sodium ophthalmic in situ gels. Int J Pharm 411(1-2): 128-135.

6. Mahmoud AA, El-Feky GS, Kamel R, Awad GE (2011) Chitosan/ sulfobutylethercyclodextrin nanoparticles as a potential approach for ocular drug delivery. Int J Pharm 413(1-2): 229-236.

7. Ammar HO, Salama HA, Ghorab M, Mahmoud AA (2009) Nanoemulsion as a potential ophthalmic delivery system for dorzolamide hydrochloride. AAPS PharmSciTech 10(3): 808-819.

8. Sultana Y, Ali A (2006) Ion-activated, gelrite-based in situ ophthalmic gels of pefloxacinmesylate: comparison with conventional eye drops. Drug Delivery 13(3): 215-219.

9. Gupta H, Aqil M, Khar RK, Ali A, Bhatnagar A, et al. (2011) Biodegradable levofloxacin nanoparticles for sustained ocular drug delivery. J Drug Targeting 19(6): 409-417.

10. Casolaro M, Casolaro I, Lamponi S (2012) Stimuli-responsive hydrogels for controlled pilocarpine ocular delivery. Eur J Pharm Biopharm 80(3): 553-561

11. adhav CM, Vaishali K, Payghan SA (2015) Formulation and Evaluation of Antifungal Non-Aqueous Microemulsion for Topical Drug Delivery of Griseofulvin. Inventi Rapid: Pharm Tech 2015(1): 1-13.

12. Ipinski CA, Lombardo F, Dominy BW, Feeney PJ (2001) Experimental and computational approaches to estimate solubility and permeability in drug discovery and development settings. Adv Drug Deliv Rev 46(13): 3-26.

13. Payghan SA, Mane YV, Kate VK, Tamboli ZJ (2015) Anhydrous Emulsion: Vehicles for Topical Delivery of Ketoconazole. Inventi Impact: NDDS 2015(1): 43-53.

14. Attar M, Shen J, Ling KH, Tang-Liu D (2005) Ophthalmic Drug Delivery Considerations at the Cellular Level: Drug-Metabolising Enzymes and Transporters. Expert Opin Drug Delivery 2(5): 891-908.

15. Midi Y, Barar J, Ahmadian S, Heidari HR, Gumbleton M, et al. (2008a) Characterization and astrocytic modulation of system $L$ transporters in brain microvasculature endothelial cells. Cell Biochem Funct 26(3): 381-391.

16. Barar J, Javadzadeh AR, Omidi Y (2008) Ocular Novel Drug Delivery: Impacts of Membranes and Barriers. Expert Opin Drug Deliv 5(5): $567-$ 581.

17. Urtti A (2006) Challenges and obstacles of ocular pharmacokinetics and drug delivery. Adv Drug Deliv Rev 58(11): 1131-1135.

18. Fischbarg J (2006) The Biology of the Eye. In: (1 ${ }^{\text {st }}$ edn), Elsevier Science, Amsterdam, Netherlands, pp. 406.

19. Freddo TF (2001) Shifting the Paradigm of the Blood-Aqueous Barrier Exp Eye Res 73(5): 581-592.

20. Conrad JM, Robinson JR (1977) Aqueous Chamber Drug Distribution Volume Measurement in Rabbits. J Pharm Sci 66(2): 219-224.

21. Anand BS, Katragadda S, Gunda S, Mitra AK (2006) In vivo ocular pharmacokinetics of acyclovir dipeptide ester prodrugs by microdialysis in rabbits. Mol Pharm 3(4): 431-440.
22. Smith M, Omidi Y, Gumbleton M (2007) Primary porcine brain microvascular endothelial cells: biochemical and functional characterization as a model for drug transport and targeting. J Drug Target 15(4): 253-268.

23. Nalavade PS, Landge KS, Kate VK, Payghan SA (2016) Effect of formulation Variables on physicochemical properties of Cholecalciferol Nonaqueous Nanoemulsion. Asian Journal of Pharmaceutics, 10(3): S357-S372.

24. Barar J, Yadollah O (2006) Nanoparticles for Ocular Drug Delivery. In: Gupta RB, Kompella UB (Eds.), Nanoparticle Technology for Drug Delivery, CRC Press, USA, pp. 287-335.

25. Kaulen P, Kahle G, Keller K, Wollensak J (1991) Autoradiographic mapping of the glucose transporter with cytochalasin B in the mammalian eye. Invest Ophthalmol Vis Sci 32(6): 1903-1911.

26. Jager K, Bonisch U, Risch M, Worlitzsch D, Paulsen F, et al. (2009) Detection and regulation of cationic amino acid transporters in healthy and diseased ocular surface. Invest Ophthalmol Vis Sci 50(3): 11121121.

27. Jentsch TJ, Janicke I, Sorgenfrei D, Keller SK, Wiederholt M, et al. (1986a) The regulation of intracellular $\mathrm{pH}$ in monkey kidney epithelial cells (BSC-1). Roles of $\mathrm{Na}+/ \mathrm{H}+$ antiport, $\mathrm{Na}+-\mathrm{HCO} 3(-)-(\mathrm{NaCO}-)$ symport, and Cl-/HCO3- exchange. J Biol Chem 261(16): 12120-12127.

28. Mitra AK, Anand BS, Duvvuri S (2006) Drug Delivery to the Eye, In: The Biology of Eye, In: Fischbarg J [Ed.], Academic Press, New York, pp. $307-$ 351.

29. Lee SS, Hughes P, Ross AD, Robinson MR (2010) Biodegradable implants for sustained drug release in the eye. Pharm Res 27(10): 2043-2053.

30. Eljarrat-Binstock E, Orucov F, Aldouby Y, Frucht-Pery J, Domb AJ, et al. (2008) Charged nanoparticles delivery to the eye using hydrogel iontophoresis. J Control Release 126(2): 156-161.

31. Higashiyama M, Tajika T, Inada K, Ohtori A (2006) Improvement of the ocular bioavailability of carteolol by ion pair. J OculPharmacolTher 22(5): 333-339.

32. Kaur IP, Smitha R (2002) Penetration enhancers and ocular bioadhesives: Two new avenues for ophthalmic drug delivery. Drug DevInd Pharm 28(4): 353-369.

33. Lang JC (1995) Ocular drug delivery conventional ocular formulation. Adv Drug Deliv Rev 16(1): 39-43.

34. Zimmer A, Kreuter J (1995) Microspheres and nanoparticles used in ocular delivery systems. Adv Drug Deliv Rev 16(1): 61-73.

35. Adibkia K, SiahiShadbad MR, Nokhodchi A, Javadzedeh A, BarzegarJalali, et al. (2007a) Piroxicam nanoparticles for ocular delivery: physicochemical characterization and implementation in endotoxin induced uveitis. J Drug Target 15(6): 407-416.

36. Adibkia K, Omidi Y, Siahi MR, Javadzadeh AR, Barzegar-Jalali M, et al. (2007) Inhibition of endotoxin-induced uveitis by methylprednisolone acetate nanosuspension in rabbits. J Ocul Pharmacol Ther 23(5): 421432 .

37. Koizumi K, Poulaki V, Doehmen S, Radetzky S, Lappas A, et al. (2003) Contribution of TNF-Alpha to leukocyte adhesion, vascular leakage, and apoptotic cell death in endotoxin-induced uveitis in vivo. Invest Ophthalmol Vis Sci 44(5): 2184-2191.

38. Pignatello R, Bucolo C, Ferrara P, Maltese A, Puleo A, et al. (2002a) Eudragit RS100® nanosuspensions for the ophthalmic controlled delivery of ibuprofen. Eur J Pharm Sci 16(1-2): 53-61.

39. Dillen K, Vandervoort J, Mooter, GVD, Ludwig A (2006) Evaluation of Ciprofloxacin-Loaded Eudragit RS100 or RL100/PLGA Nanoparticles. Int J Pharm 314(1): 72-82. 
40. Pignatello R, Ricupero N, Bucolo C, Maugeri F, Maltese A, et al. (2006) Preparation and characterization of eudragit retard nanosuspensions for the ocular delivery of cloricromene. AAPS PharmSciTech 7(1): E27.

41. Marchal-Heussler L, Sirbat D, Hoffman M, Maincent P (1993) Poly( $\varepsilon$ caprolactone) nanocapsules in carteolol ophthalmic delivery. Pharm Res 10(3): 386-390.

42. Zimmer AK, Serbe H, Kreuter J (1994) Evaluation of pilocarpine-loaded albumin particles as drug delivery systems for controlled delivery in the eye, I: in vitro and in vivo characterizat on. J Control Release 32(1): 57-70.

43. Calvo P, Vila-Jato JL, Alonso MJ (1997) Evaluation of cationic polymer coated nanocapsules as ocular drug carriers. Int J Pharm 153(1): 4150 .

44. Pignatello R, Bucolo C, Ferrara P, Maltese A, Puleo A, et al. (2002a) Eudragit RS100® nanosuspensions for the ophthalmic controlled delivery of ibuprofen. Eur J Pharm Sci 16(1-2): 53-61.

45. Perumal D, Dangor CM, Alcock RS, Hurbans N, Moopanar KR, et al (1999) Effect of formulation variables on in vitro drug release and micromeritic properties of modified release ibuprofen microspheres J Microencapsul 16(4): 475-487.

46. Pignatello R, Chiechio SAD, Giunchedi P, Spadaro C, Puglisi G, et al. (2001) Preparation and analgesic activity of Eudragit RS100 microparticles containing diflunisal. Drug Deliv 8(1): 35-45

47. B"ohm BHL, M"uller RH (1999) Lab-scale production unit design for nanosuspensions of sparingly soluble cytotoxic drugs. Pharm Sci Tech Today 2(8): 336-339.

48. Baravkar VS, Kate VK, Jirage AS, Payghan SA (2014) Potential application of non Aqueous emulsion for drug delivery. Asian Journal of Biomedical and Pharmaceutical Sciences 4(29): 10-18.

49. Bucolo C, Maltese A, Puglisi G, Pignatello R (2002) Enhanced ocular anti-inflammatory activity of ibuprofen carried by a Eudragit RS100® nanoparticle suspension. Ophthalmic Res 34(5): 319-323.
50. Cunha-Vaz JG (2006) The Blood-Ocular Barriers: Past, Present, and Future. Doc Ophthalmol 93(1-2): 149-157.

51. Duvvuri S, Majumdar S, Mitra AK (2004) Role of Metabolism in Ocular Drug Delivery. Curr Drug Metab 5(6): 507-515.

52. Hamsika M, Gowda DV, Jigyasa V, Afrasim M (2016) Nanotechnology for Ophthalmic Preparations. Int J Curr Pharm Res 8(2): 5-11.

53. Jadhav CM, Kate VK, Payghan SA, Disouza JI (2014) Investigating Application of Non Aqueous Microemulsion for Drug Delivery. Asian Journal of Biomedical and Pharmaceutical Sciences 4(29): 1-9.

54. Jadhav CM, Kate V, Payghan SA (2015) Formulation and Evaluation of Antifungal Non-Aqueous Microemulsion for Topical Drug Delivery of Griseofulvin. Inventi Impact: Pharm Tech 2015(1): 38-50.

55. Jadhav CM, Kate V, Payghan SA (2015) Investigation of effect of non-ionic surfactant on preparation of griseofulvin non-aqueous nanoemulsion. Journal of Nanostructure in Chemistry (Springerlink com) 5(1): 107-113.

56. Jadhav CM, Kate VK, Payghan SA (2014) Stability Study of Griseofulvin in Non Aqueous Microemulsion System. Asian Journal of Biomedical and Pharmaceutical Sciences 4(35): 79-83.

57. Maryam A, Byrne ME (2008) Challenges and solutions in topical ocular drug-delivery systems. Expert Rev Clin Pharmacol 1(1): 145-161.

58. Payghan SA, Purohit SS, Shrivastava DN (2008) Non-aqueous emulsion: versatile vehicle for drug delivery. Pharmaceutical Reviews.

59. Payghan SA, Shrivastava DN Potential of solubility in drug discovery and development. Pharmaceutical Reviews.

60. Pignatello R, Amico D, Chiechio S, Giunchedi P, Spadaro C, et al. (2001) Preparation and analgesic activity of Eudragit RS100 microparticles containing diflunisal. Drug Deliv 8(1): 35-45.

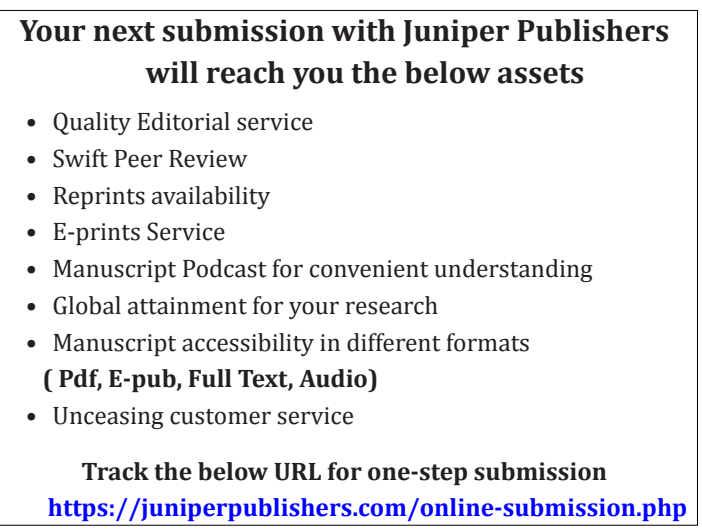

